\title{
Letter to the editor concerning first-line therapy with afatinib - an irreversible EGFR TKI and overall survival of NSCLC patients with EGFR gene mutations
}

\author{
Paweł Krawczyk, Tomasz Powrózek, Marcin Nicoś, Janusz Milanowski \\ Department of Pneumonology, Oncology and Allergology, Medical University of Lublin, Poland
}

Contemp Oncol (Pozn) 2015; 19 (3): 250-251

DOI: $10.5114 /$ wo.2015.52660

We have read with great attention the recent manuscript by James Yang and co-workers published in Lancet Oncology [1]. The authors had reported positive effect of I-line afatinib treatment on overall survival (OS) of lung adenocarcinoma patients with EGFR gene mutations through an analysis of data from two open label, randomized, phase 3 trials: LUX-Lung 3 and LUX-Lung 6. In both clinical trials, the patients with exon 19 deletions of EGFR gene had shown significantly longer OS when therapy strategy was started from I-line afatinib than from I-line chemotherapy (31.7 vs. 20.7 months). By contrast, OS of patients with L858R substitution in exon 21 of EGFR gene was insignificantly longer in the chemotherapy arm in comparison to the afatinib arm (22.1 vs. 26.9 months). However, progression free survival (PFS) in afatinib group was significantly longer than in chemotherapy group irrespective of EGFR mutation type.

In previous clinical trials, significant differences in overall survival between patients received I-line EGFR tyrosine kinase inhibitors (EGFR TKIs) and I-line chemotherapy have not been reported, primarily because most patients in progression after I-line chemotherapy were subsequently treated with EGFR TKIs. However, patients progressing during EGFR TKIs treatment rarely continued EGFR TKIs therapy beyond RECIST progression.

Therefore, the question arises whether the groups of patients with exon 19 deletion and L858R substitution in LUX-Lung 3 and 6 clinical trials were well matched? In whole studied group, $73.8 \%$ patients with exon 19 deletion and $69.7 \%$ patients with exon 21 substitution received subsequent systemic therapies after study treatment discontinuation. However, it is not explained how many lines of therapy were applied and which treatment regiments were used in each line.

In LUX-Lung 6 trial, in afatinib arm, subsequent treatment with EGFR TKIs received 36 patients (33\%) with exon 19 deletion, while only 14 patients (17\%) with L858R substitution. It can be assumed that these patients had a EGFR TKIs therapy beyond RECIST progression, after which, in clinical progression, the chemotherapy could be used. One could speculate that, in chemotherapy arm, EGFR TKIs therapy was administrated insignificantly rare in patients with exon 19 deletion (53\%) than in patients with L858R substitution (61\%). If this is true?

Information about the subsequent systemic therapy of patients in LUX-Lung 3 clinical trial are very obscure. The authors shown (Table 2) that, in different treatment arms, the subsequent therapy received $110-126 \%$ of patients. It is obvious that some of the patients received additionally two or three lines of treatment (chemotherapy and EGFR TKIS). The question is what percentage of patients with exon 19 deletion and L858R substitution were treated with II-line EGFR TKIs therapy after I-line afatinib treatment beyond RECIST progression?

It has been suggested that EGFR TKIs therapy continuation even after disease progression may improve overall survival compared with chemotherapy in NSCLC patients harboring activating EGFR gene mutations. Whereas disease flare was reported after sudden EGFR TKIs discontinuation [2]. Clinical trials with continued erlotinib, gefitinib or afatinib are in progress [3-5]. Therefore, it seems that a higher percentage of patients treated with EGFR TKIs beyond RECIST progression in exon 19 deletion group than in L858R substitution group can affect the differences in OS.

The authors declare no conflict of interest.

\section{References}

1. Yang JCH, Wu YL, Schuler M, et al. Afatinib versus cisplatin-based chemotherapy for EGFR mutation-positive lung adenocarcinoma (LUX-Lung 3 and LUX-Lung 6): analysis of overall survival data from two randomized, phase 3 trials. Lancet Oncology 2015; http://dx. doi.org/10.1016/S1470-2045(14)71173-8.

2. Chaft JE, Oxnard GR, Sima CS, et al. Disease flare after tyrosine kinase inhibitor discontinuation in patients with EGFR-mutant lung cancer and acquired resistance to erlotinib or gefitinib: implications for clinical trial design. Clin Cancer Res 2011; 17: 6298-6303.

3. Park K, Tsai C-M, Ahn M-J, et al. ASPIRATION: Phase II study of continued erlotinib beyond RECIST progression in Asian patients (pts) with epidermal growth factor receptor (EGFR) mutation-positive non-small cell lung cancer (NSCLC). J Clin Oncol 2012; 30: abstr. TPS7614.

4. Mok TS, Wu Y, Nakagawa K, et al. Gefitinib/chemotherapy vs chemotherapy in epidermal growth factor receptor (EGFR) muta- 
tion-positive non-small-cell lung cancer (NSCLC) after progression on first-line gefitinib: the Phase III, randomised IMPRESS study. Ann Oncol 2014; 25 (Suppl 5): v1-v41, doi:10.1093/annonc/mdu438.45.

5. Schuler MH, Yang CH, Park K, et al. Continuation of afatinib beyond progression: Results of a randomized, open-label, phase III trial of afatinib plus paclitaxel versus investigator's choice chemotherapy in patients with metastatic non-small cell lung cancer progressed on erlotinib/gefitinib (E/G) and afatinib - LUX-Lung 5. J Clin Oncol 2014; 32(5s): abstr. 8019

\section{Address for correspondence}

\section{Paweł Krawczyk}

Jaczewskiego 8

20-954 Lublin, Poland

e-mail: krapa@poczta.onet.pl

Submitted: 16.03 .2015

Accepted: 8.04 .2015 